\section{An Unusual Cause of Recurrent Laryngeal Nerve Monitoring Failure during Parathyroidectomy} KATARINA BOJANIĆ • AMANDA R. MORASKA • JURAJ SPRUNG・
GEOFFREY B. THOMPSON • TOBY N. WEINGARTEN

TOBY N. WEINGARTEN $(\bowtie)$ Assistant Professor of Anesthesiology From the Department of Anesthesiology and Department of Surgery College of Medicine, Mayo Clinic, 200 First Street SW, Rochester, MN 55905

Phone: (507) 255-1612

Fax: (507) 255-6463

E-mail: weingarten.toby@mayo.edu.

KATARINA BOJANIĆ Resident in Pediatrics (Research Trainee at Mayo Clinic)

AMANDA R. MORASKA Mayo Clinic Medical Student

JURAJ SPRUNG Professor of Anesthesiology

GEOFFREY B. THOMPSON Professor of Surgery

\begin{abstract}
Intraoperative monitoring of the recurrent laryngeal nerve $(R L N)$ during surgical dissection allows for real time identification and assessment of nerve function integrity. Since neuromuscular blockade interferes with electromyography, long-acting muscle relaxants cannot be used during anesthesia. We report a patient in whom monitoring of the RLN was unsuccessful because of prolonged muscle paralysis following the administration of succinylcholine, presumably due to a pseudocholinesterase deficiency.
\end{abstract}

Key words: laryngeal nerve monitoring, muscle paralysis, pseudocholinesterase deficiency

\section{Introduction}

Intraoperative monitoring of the recurrent laryngeal nerve (RLN) with electromyography improves precision in identification of the RLN and provides immediate electromyographic feedback regarding its integrity during surgical dissection. A method of intraoperative $R L N$ monitoring involves the use of electrodes attached to the surface of an endotracheal tube that are placed in contact with the vocal cords during tracheal intubation. (1) Since neuromuscular blockade interferes with electromyography, long-acting nondepolarizing muscle relaxants cannot be used when this type of monitoring is employed. Rather, only succinylcholine is used to facilitate tracheal intubation. We report a patient in whom intraoperative monitoring of the RLN was unsuccessful because of unrecognized prolonged muscle paralysis following the administration of succinylcholine, presumably due to one of the variants of pseudocholinesterase (PsChE) deficiency.

\section{Case report}

Permission for this single case was waived by the Mayo Clinic, Rochester MN, Institutional Review Board. A 53-year old, female (height: $170 \mathrm{~cm}$, weight: $64.9 \mathrm{~kg}, \mathrm{BMl}: 22.4 \mathrm{~kg} / \mathrm{m}^{2}$ ) with primary hyperparathyroidism was admitted for resection of parathyroid adenoma due to primary hyperparathyroidism (serum calcium $11.1 \mathrm{mg} / \mathrm{dL}$ and parathyroid hormone concentration $96 \mathrm{pg} / \mathrm{mL}$ with a focus of discordant sestamibi uptake in the left thyroid bed compatible with a parathyroid adenoma). Except for the history of nephrolithiasis she was in an excellent state of health and was taking no medications.

Her preanesthetic evaluation was unremarkable except for her report that she was "slow to recovery" from anesthesia following an inguinal hernia repair 15 years ago. She was unable to give any specific information regarding adverse perioperative events during that surgery. General anesthesia was induced with $150 \mathrm{mg}$ propofol i.v. and $100 \mathrm{mcg}$ fentanyl i.v. Endotracheal intubation was facilitated with 100 mg succinylcholine i.v. (during parathyroid surgery and after a single dose of succinylcholine we do not conduct routine monitoring 
of neuromuscular blockade with peripheral nerve stimulator, because arms are tucked [ulnar nerve monitoring] and head is covered with drapes [orbicularis oculi monitoring]). The endotracheal tube used was a $7.0 \mathrm{~mm}$ diameter Sheridan ${ }^{\circledR}$ tube (Mallinckrodt, Hudson, $\mathrm{RCl}$ ) with the recording single channel surface laryngeal electrode (Dragonfly ${ }^{\circledR}$, Neurovision Medical products, INC, Ventura, Ca, USA) attached circularly $2 \mathrm{~cm}$ above the cuff with placement between the vocal cords. The Nerveäna Surgical Nerve Locator (Neurovision Medical products, INC, Ventura, Ca, USA) system was used to monitor laryngeal nerves. After positioning for surgery with neck extension, the proper position of the electrodes in relation to the vocal cords was reconfirmed under visualization with direct laryngoscopy. Anesthesia was maintained with a combination of a propofol infusion $(120 \mu \mathrm{g} /$ $\mathrm{kg} / \mathrm{min}$ ) and desflurane $2-4 \%$.

Following neck incision, the carotid sheath and RLN were visually identified, and the Nerveäna proactive locator (an insulated spatula) was used to stimulate the left vagus and left RLN nerve in order to elicit an audible electromyographic (EMG) signal. The surgeon increased stimulation intensity amplitude gradually from $0.5 \mathrm{~mA}$ to the maximum allowable amplitude of 3.0 $\mathrm{mA}$. Stimulation signals were at preset settings of $4 \mathrm{~Hz}$ with 150 microsecond pulses. In this case direct stimulation failed to elicit any audible EMG signal even at the maximum amplitude, nor we obtained a visible compound action potential waveform (figure 1). Finally, there was no visual evidence of contraction of the posterior cricoarytenoid muscle. Thus, nerve conduction integrity could not be assessed with this monitor. Because the most frequent cause of a nonconducting signal is malposition of the surface electrodes in relation to vocal cords, the anesthesiologist again ensured proper placement of the surface electrodes against the vocal cords with direct laryngoscopic visualization (McGRATH® Video Laryngoscope). All monitoring system connections were re-inspected and appeared

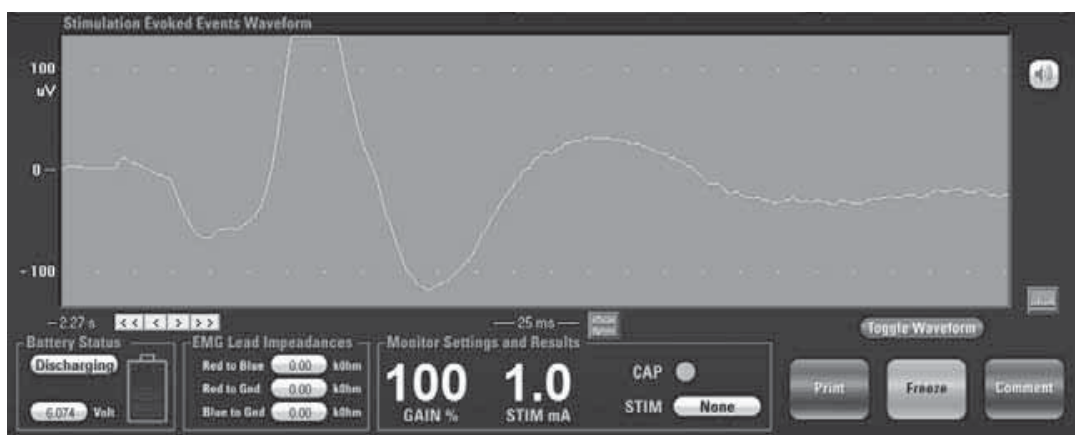

Figure 1. Stimulation of recurrent laryngeal nerve with Nerveäna proactive locator elicited an EMG signal. Typically, stimulation intensity amplitude is increased gradually from $0.5 \mathrm{~mA}$ to the maximum allowable amplitude of 3.0 mA. Absence of successful stimulus response to elicit a robust action potential (in a form of an EMG waveform) indicates either presence of a technical problem (vocal cords are not in contact with electrodes), accidentally transected nerve (surgical injury), or presence of muscle paralysis (use of nondepolarizing muscle relaxants or in our case, the use of succinylcholine in a patient with pseudocholinesterase deficiency).

intact. The monitoring system continued to fail to detect nerve conduction and the parathyroidectomy continued without the assistance of nerve monitoring utilizing standard direct visualization of RLN. The surgery lasted 90 minutes, which included waiting time for intraoperative parathyroid hormone (PTH) measurement, during which time the patient was kept asleep with propofol (25 $\mu \mathrm{g} / \mathrm{kg} / \mathrm{min})$. Desflurane was discontinued 30 minutes before the end of surgery. The total amount of analgesics administered during the surgery was $200 \mathrm{mcg}$ fentanyl and $15 \mathrm{mg}$ of ketorolac. Intraoperative PTH concentration fell to greater than $50 \%$ of baseline levels and the patient was deemed cured. After obtaining these results the propofol was discontinued. Blood pressure increased from $110 / 75 \mathrm{mmHg}$ to $165 / 90 \mathrm{mmHg}$, and heart rate from 75 bpm to $135 \mathrm{bpm}$. However, the patient did not move or show signs of respiratory effort. A peripheral nerve stimulator was placed on the ulnar nerve to assess muscle function, and using the trainof-four (TOF) stimulation mode elicited only 1 faint twitch of adductor pollicis brevis muscle. Tetanic stimulation at 50 $\mathrm{Hz}$ showed a fade pattern of adductor pollicis brevis. This suggested generalized muscle paralysis in our patient who did not receive a nondepolarizing muscle relaxant. Therefore, in order to prevent awareness, $60 \mathrm{mg}$ of propofol was administered followed by a propofol infusion $(45 \mu \mathrm{g} / \mathrm{kg} / \mathrm{min})$. After an additional 25 minutes, the patient regained only 2 faint TOF twitches. Over the next 45 minutes, she regained all 4 TOF twitches and full muscle strength (5 seconds of sustained head lift). She was allowed to awaken and her trachea was extubated without further incident. Since this clinical picture was consistent with PsChE deficiency, appropriate laboratory studies were obtained: total PsChE activity was $1681 \mathrm{U} / \mathrm{L}$ (normal, $2550-6800 \mathrm{U} / \mathrm{L}$ ) and dibucaine number was 83\% (reference range $70-90 \%$ inhibition).

\section{Discussion}

We described a patient in whom intraoperative monitoring of RLN was not possible due to prolonged muscle blockade following a single dose of succinylcholine, which was presumably caused by slowed metabolism of succinylcholine due to PsChE deficiency. Presence of the fade on the TOF stimulation after succinylcholine in our patient suggests a phase II block or desensitizing block, which is consistent with prolonged succinylcholine metabolism in patients with PsChE deficiency. $(2,3)$ In the presence of muscle paraly- 
sis, no response or a weak response would be unlikely to trigger the audible alarm on EMG monitoring device used on our patient.

The inability to monitor the RLN with electromyography triggered initially a search for mechanical failure (i.e., malposition of the endotracheal tube electrodes), but the cause was not elucidated until the end of surgery when we noticed that the patient had profound muscle paralysis which was clinically consistent with PsChE deficiency. Use of peripheral nerve stimulator earlier during the case could have helped to diagnose the problem, but this was not done. While we routinely use peripheral nerve stimulators in majority of our cases where non-depolarizing muscle relaxants are administered, these monitors are not typically employed following the administration of a single dose of succinylcholine to facilitate tracheal intubation and when no other muscle relaxants will be used. PsChE deficiency, especially the homozygous type, is a relatively rare condition, and we did not consider it in differential diagnosis after we encountered problems in RLN monitoring in our patient.
In our patient, the result of testing for the most common types of PsChE deficiency (silent gene, and/or dibucaine resistant variant) rendered the precise diagnosis of the variant of PsChE deficiency inconclusive. The patient's total pseudocholinesterase activity was 35\% below the lower limit of normal, and the dibucaine inhibition was normal. These tests excluded the most frequent mutations of the butyrylcholinesterase gene (gene responsible for PsChE deficiency): homozygous A-variant [AA] or silent (S) mutation [SS], or a combination of an A-variant and a (S) mutation [AS]. In these genotypes the dibucaine number would be reduced to a value of 10-30\% inhibition of PsChE, and/or total PsChE activity would be low. However, there are numerous, but rare mutations of the butyrylcholinesterase gene, which are not tested within the routine clinical setting, for example the $\mathrm{K}$ variant which is identified only with DNA sequencing. (4) Another abnormal genotype is a fluoride resistant butyrylcholinesterase gene, i.e., the fluoride resistant PsChE variant (homozygous variant present in 1/150.000). (5) Homozygous patients with the fluoride resistant gene would be expected to have a paralysis after succinylcholine which ranges from 1 to 2 hours, (6) which correspond to muscle paralysis encountered in our patient. In our institution, as is true for clinical institutions across the United States, testing for rare mutations in the butyrylcholinesterase gene is not performed. Therefore, we cannot comment on the exact etiology of the prolonged neuromuscular blockade to succinylcholine in our patient. Our patient did not have any other conditions which could lead to prolongation of succinylcholine action (certain tumors, use of medication such as timolol, cyclophosphamide, bambuterol, aminoglycosides, neostigmine). (6)

In conclusion, we have described an unusual case of inability to monitor the RLN with EMG in a patient who was fully paralyzed after succinylcholine presumably due to PsChE deficiency. In cases of unexplained inability to monitor the RLN, anesthesiologists should expand the differential diagnosis beyond the most typical malfunction faults (equipment malfunction) and should evaluate potential for other rare conditions such as one described in our patient.

\section{REFERENCES}

1. Hemmerling TM, Schmidt J, Bosert C, Jacobi KE, Klein P. Intraoperative monitoring of the recurrent laryngeal nerve in 151 consecutive patients undergoing thyroid surgery. Anesth Analg 2001;93:396-9.

2. Harada T, Shimonaka H, Yamamoto M, Akamatsu S, Kasamatsu M, Gotoh M, et al. Succinylcholine neuromuscular blockade in a case of pseudocholinesterase variant. Masui 1989;38:791-6.

3. Viby-Mogensen J. Succinylcholine neuromuscular blockade in subjects homozygous for atypical plasma cholinesterase. Anesthesiology 1981;55:429-34.

4. Whittaker M. Plasma cholinesterase variants and the anaesthetist. Anaesthesia 1980;35:174-97.

5. Harris $\mathrm{H}$, Whittaker M. Differential inhibition of human serum cholinesterase with fluoride: recognition of two new phenotypes. Nature 1961;191:496-8.

6. Soliday FK, Conley Y, P., Henker R. Pseudocholinesterase deficiency; A comprehensive review of genetic, acquired, and drug influences. AANA Journal 2010;78:313-20. 